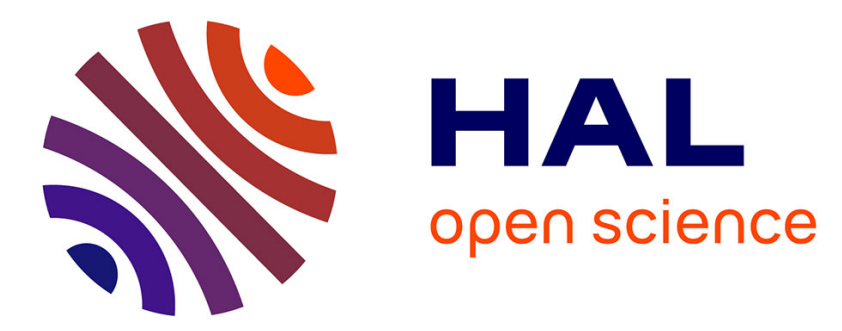

\title{
Modulation spatio-temporelle en spectroscopie des jets moléculaires. Déplacement radiatif
}

\author{
J.C. Chardon, C. Genty, J.J. Miller, J.G. Theobald
}

\section{To cite this version:}

J.C. Chardon, C. Genty, J.J. Miller, J.G. Theobald. Modulation spatio-temporelle en spectroscopie des jets moléculaires. Déplacement radiatif. Revue de Physique Appliquée, 1979, 14 (5), pp.649-652. 10.1051/rphysap:01979001405064900 . jpa-00244643

\section{HAL Id: jpa-00244643 https://hal.science/jpa-00244643}

Submitted on 1 Jan 1979

HAL is a multi-disciplinary open access archive for the deposit and dissemination of scientific research documents, whether they are published or not. The documents may come from teaching and research institutions in France or abroad, or from public or private research centers.
L'archive ouverte pluridisciplinaire HAL, est destinée au dépôt et à la diffusion de documents scientifiques de niveau recherche, publiés ou non, émanant des établissements d'enseignement et de recherche français ou étrangers, des laboratoires publics ou privés. 


\title{
Modulation spatio-temporelle en spectroscopie des jets moléculaires. Déplacement radiatif
}

\author{
J. C. Chardon, C. Genty, J. J. Miller et J. G. Theobald \\ Laboratoire de Spectroscopie Hertzienne et d'Electronique, Faculté des Sciences, 25030 Besançon, France
}

(Reçu le 7 décembre 1978, révisé le 5 février 1979, accєt 'é le 6 février 1979)

\begin{abstract}
Résumé. - Dans un article précédent [1] nous avons étudié par une théorie de perturbations au 1 er ordre les phénomènes se produisant au voisinage d'une résonance lorsque le champ d'irradiation présente une structure spatiale périodique. En utilisant un modèle différent, mais permettant un calcul exact de la probabilité de transition, nous retrouvons les résultats antérieurs et rendons compte d'un déplacement radiatif mis expérimentalement en évidence.
\end{abstract}

\begin{abstract}
In a preceding paper we have used the first order perturbation theory to describe the phenomena occurring near a molecular resonance when the irradiation field presents a spatial periodic structure. Using a different model allowing an exact calculation we find again the preceding results and also a new type of radiative shift which we have observed.
\end{abstract}

1. Introduction. - Les expériences sont réalisées avec un spectromètre à jet de méthanal du type Rabi à détection par le jet. Le système de modulation spatio-temporel est celui décrit en référence [1]. Nous avions alors utilisé des cellules à $n$ éléments, constituées soit de barres cylindriques, soit de condensateurs plans et fait, dans un cas comme dans l'autre, une approximation [2] de champ modulé suivant un rythme sinusoïdal. De telles modulations spatiotemporelles ont déjà été observées par Hadeishi et coll. [3] d'une part, Pommier et Shermann [4] d'autre part, qui utilisent la modulation résonnante d'un champ continu créée par le mouvement des particules pour produire une résonance magnétique.

Dans le cas de cellules à condensateurs, on peut penser utiliser un modèle différent et faire l'approximation d'un champ R.F. modulé en créneau. Les expériences concernant cet article ont été réalisées avec un système à 4 éléments $(n=4)$ représenté dans le montage expérimental de la figure 1 . C'est pour ce nombre $n$ que les expériences ont donné les résultats les plus facilement exploitables, tant du point de vue expérimental que théorique. En effet, le dispositif expérimental approche d'autant mieux le modèle théorique choisi (cf. ci-dessous) que le nombre d'éléments est grand, les effets de bord à l'entrée et à la sortie de la cellule prenant une importance relative moindre. Par contre, le procédé itératif employé rend le calcul de la probabilité de transition de plus en plus long et complexe au fur et à mesure que le nombre $n$ augmente. Le choix de $n=4$ est donc un compromis entre ces deux impératifs contradictoires. Une série d'expériences avec une cellule de 26 éléments à barres cylindriques (décrite et utilisée en référence [1]) a été reprise en vue de comparer les déplacements radiatifs pour des $n$ très différents.

Le champ vu par une molécule présente une amplitude variant en créneau avec inversion de phase quand on passe d'un élément au suivant. Bien que ce modèle théorique comporte toujours une approximation dans le fait que le champ réel ne présente pas de discontinuités franches à l'entrée et à la sortie des éléments, il offre l'avantage essentiel de permettre un calcul exact de la probabilité de transition, selon la méthode de Ramsey [7].

2. Calcul de la probabilité de transition. - Le dispositif d'irradiation crée pour une molécule un champ qui évolue en fonction du temps comme le montre la figure 2: La molécule est irradiée pendant un temps $T$, par un champ d'amplitude constante, puis traverse une région de champ nul pendant le même temps $T$, et ainsi de suite, la phase du champ s'inversant quand on passe d'un élément au suivant.

La fonction d'onde s'écrit :

$$
|\psi\rangle=c_{1}(t)\left|\varphi_{1}\right\rangle+c_{2}(t)\left|\varphi_{2}\right\rangle
$$

(notations de la référence [1]).

Les coefficients

$$
c_{1}(t)=b_{1}(t) \mathrm{e}^{-i \omega_{1} t}
$$

et

$$
c_{2}(t)=b_{2}(t) \mathrm{e}^{-i \omega_{2} t}
$$




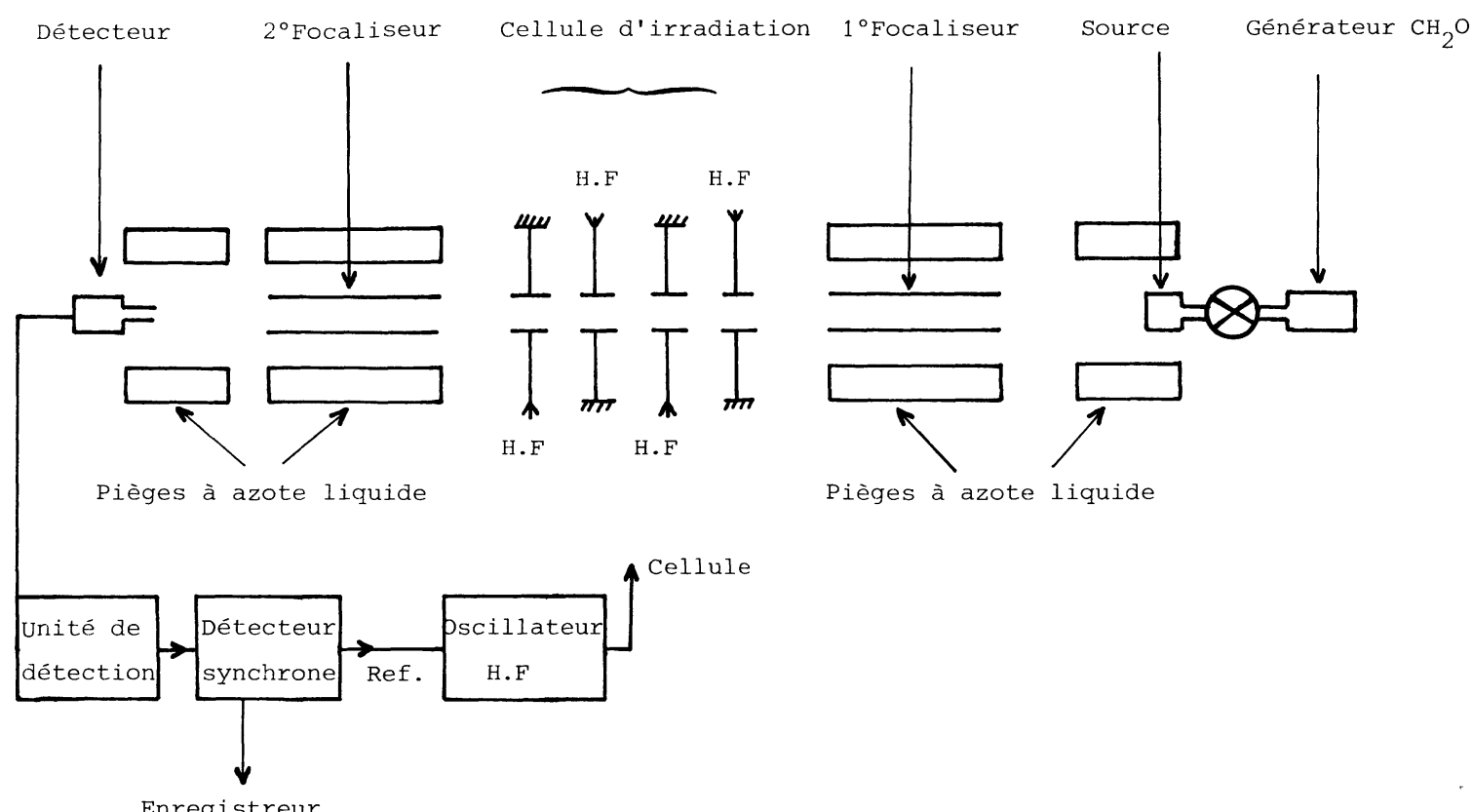

Fig. 1. - Schéma du spectromètre avec la cellule de modulation spatio-temporelle. L'appareillage est décrit en références [5] et [6]. [Diagram of the spectrometer with the spatio-temporal modulation cell. The apparatus is described in references [5] and [6].]

évoluent entre les instants $t_{0}$ et $t_{0}+t$ suivant [7] :

$$
\begin{aligned}
& c_{1}\left(t_{0}+t\right)=\left[A c_{1}\left(t_{0}\right)-C \mathrm{e}^{-i\left(\omega t_{0}+\varphi\right)}\right] \exp \left[-i\left(\frac{\Delta}{2}+\omega_{1}\right) t\right] \\
& c_{2}\left(t_{0}+t\right)=\left[B c_{2}\left(t_{0}\right)+C \mathrm{e}^{i\left(\omega t_{0}+\varphi\right)}\right] \exp \left[i\left(\frac{\Delta}{2}-\omega_{2}\right) t\right]
\end{aligned}
$$

où $\varphi$ est la phase du champ $(\varphi=(n-1) \pi$ sur le $n$-ième élément), $\Delta$ est l'écart à la résonance et

$$
\begin{aligned}
& A=\cos \frac{\gamma}{2} t+i \frac{\Delta}{\gamma} \sin \frac{\gamma}{2} t \\
& B=A^{*} \\
& C=2 \frac{\beta}{\gamma} \sin \frac{\gamma}{2} t \\
& \gamma=\left[\Delta^{2}+4 \beta^{2}\right]^{1 / 2} .
\end{aligned}
$$

Avec la notation $c_{i p}=c_{i}(p T)$ on obtient les formules de récurrence suivantes qui permettent de calculer les $c_{i p}$ de proche en proche :

$$
\begin{aligned}
& c_{1 p}=c_{1, p-1} \mathrm{e}^{-i \omega_{1} T} \\
& c_{2 p}=c_{2, p-1} \mathrm{e}^{-i \omega_{2} T}
\end{aligned}
$$

pour $p$ pair, c'est-à-dire pour les régions de champ nul et

$$
\begin{aligned}
& c_{1, p}=\left[A c_{1, p-1}-C \mathrm{e}^{-i[(p-1) \omega T+\varphi]} c_{2, p-1}\right] \exp \left[-i\left(\frac{\Delta}{2}+\omega_{1}\right) T\right] \\
& c_{2, p}=\left[B c_{2, p-1}+C \mathrm{e}^{i[(p-1) \omega T+\varphi]} c_{1, p-1}\right] \exp \left[i\left(\frac{\Delta}{2}-\omega_{2}\right) T\right]
\end{aligned}
$$

pour $p$ impair, c'est-à-dire pour les régions de champ R.F. d'amplitude constante.

On s'intéresse à la probabilité de transition, soit $\left|c_{2, p}\right|^{2}$ à la sortie de chaque élément, donc uniquement aux $p$ impairs. Ainsi, en remplaçant dans (5) les $c_{i, p-1}$ par leur expression (4) on obtient, pour $p$ impair uniquement $(p>1)$ :

$$
\begin{aligned}
& c_{1 p}=\left[A c_{1, p-2}-C \mathrm{e}^{-i\left[(p-1) \omega T-\omega_{12} T+\varphi\right]} c_{2, p-2}\right] \exp \left[-i\left(\frac{\Delta}{2}+2 \omega_{1}\right) T\right] \\
& c_{2 p}=\left[B c_{2, p-2}+C \mathrm{e}^{i\left[(p-1) \omega T-\omega_{12} T+\varphi\right]} c_{1, p-2}\right] \exp \left[i\left(\frac{\Delta}{2}-2 \omega_{2}\right) T\right]
\end{aligned}
$$




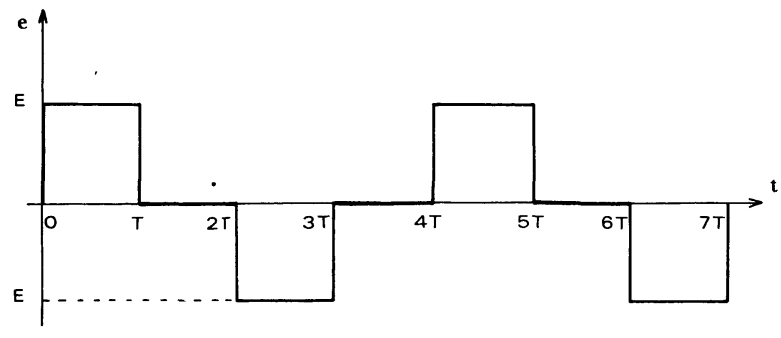

Fig. 2. - Configuration du champ vu par une molécule. [Configuration of the field for a moving molecule.]

expressions qui permettent de calculer la probabilité de transition $\left|c_{2 p}\right|^{2}$ à la sortie de chaque élément :

$$
\begin{aligned}
& \text { 1er élément }:\left|c_{21}\right|^{2}=4 \frac{\beta^{2}}{\gamma^{2}} \sin ^{2} \frac{\gamma}{2} T \\
& \text { 2e élément }:\left|c_{23}\right|^{2}=4 C^{2} N^{2} \\
& \text { 3e élément }:\left|c_{25}\right|^{2}=C^{2}\left[1+8 N^{2}\left(2 N^{2}-1\right)\right] \\
& 4^{e} \text { élément }:\left|c_{27}\right|^{2}=\left[4 C N\left(2 N^{2}-1\right)\right]^{2}
\end{aligned}
$$

avec

$$
N=\cos \frac{\gamma}{2} T \sin \frac{\Delta}{2} T+\frac{\Delta}{\gamma} \sin \frac{\gamma}{2} T \cos \frac{\Delta}{2} T .
$$

3. Résultats. - Le calcul précédent de la probabilité de transition $P(\Delta)=\left|c_{27}\right|^{2}$ suppose un jet monocinétique et un élément de matrice $\beta$ unique.

Dans nos conditions expérimentales, nous avons affaire à une distribution de vitesses avec détente $[1,8]$ décrite par la relation :

$$
J(v)=\frac{2 v^{3}}{\alpha^{4}} \exp \left[-\left(\frac{v-U}{\alpha}\right)^{2}\right]
$$

donc la probabilité de transition s'écrit :

$$
\begin{aligned}
P(\Delta)=k \frac{32}{\alpha^{4}} \int_{0}^{\infty}\left[C N\left(2 N^{2}-1\right)\right]^{2} v^{3} \times \\
\times \exp \left[-\frac{(v-U)^{2}}{\alpha^{2}}\right] \mathrm{d} v .
\end{aligned}
$$

Le coefficient de proportionnalité $k$ résulte du fait que l'intégrale $\int_{0}^{\infty} J(v) \mathrm{d} v$ n'est pas normalisée.

La courbe 1 de la figure 3 donne l'évolution de la probabilité de transition en fonction de $\Delta$, pour $\beta=2 \mathrm{kHz}$ (correspondant à un champ R.F. de $8,7 \mathrm{mV} / \mathrm{cm}$ ). La longueur $l$ de chaque élément est de $1,5 \mathrm{~cm}, U=630 \mathrm{~m} / \mathrm{s}$ et $\alpha=155 \mathrm{~m} / \mathrm{s}$. On note que cette théorie nous conduit bien à un spectre de même allure que ceux obtenus par la théorie des perturbations au $1^{\text {er }}$ ordre : Existence de 2 raies principales et de 2 raies secondaires à l'intérieur de celles-ci. On voit en outre apparaître des composantes extérieures.

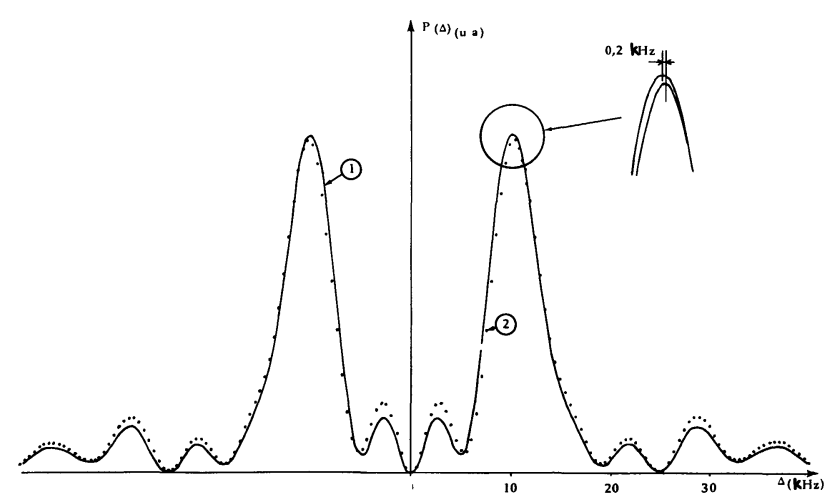

Fig. 3. - Spectres théoriques : Courbe $1:$ Spectre avec $\beta$ unique. Courbe 2: Spectre avec dégénérescence en $M$.

[Theoretical spectra : Curve 1 : Spectrum with single $\beta$. Curve 2 : Spectrum with $M$ degeneracy.]

Par ailleurs, nos expériences étant réalisées sur la transition $4_{3}$ du méthanal, il existe de surcroît une dégénérescence en $M$; l'élément de matrice $\beta$ n'est donc pas unique, mais comporte 4 termes si bien que la probabilité de transition est en fait la somme

$$
P(\Delta)=\frac{1}{4} \sum_{M=0}^{4} P_{M}(\Delta)
$$

avec

$$
\begin{aligned}
& P_{M}(\Delta)=k \cdot \frac{32}{\alpha^{4}} \int_{0}^{\infty}[C N(2\left.\left.N^{2}-1\right)\right]_{M}^{2} v^{3} \times \\
& \times \exp \left[-\left(\frac{v-U}{\alpha}\right)^{2}\right] \mathrm{d} v
\end{aligned}
$$

l'indice $M$ signifiant que dans les expressions $\beta$ est remplacé par $\beta_{M}$, élément de matrice associé à l'état $M$. (Pour la transition $4_{3}$ considérée, $\beta_{M}=0,087 \mathrm{ME}$, $\beta_{M}$ en $\mathrm{kHz}, E$ en $\mathrm{mV} / \mathrm{cm}$.)

La courbe en pointillés sur la figure 3 représente le spectre théorique obtenu dans les mêmes conditions que la courbe 1, avec un champ R.F. d'amplitude $8,7 \mathrm{mV} / \mathrm{cm}$, mais en considérant les $4 \beta_{M}$; On constate que les deux spectres ont la même allure, la position des raies principales étant cependant légèrement différente (écart de $0,4 \mathrm{kHz}$ ).

Cet écart résulte précisément de l'effet nouveau que permet de mettre en évidence cette théorie, à savoir le déplacement radiatif. Les courbes de la figure 4 donnent le déplacement radiatif théorique d'une raie principale (position $x_{M}$ d'une composante principale par rapport à la fréquence centrale) dans l'approximation de $\beta$ unique d'une part (courbe 1) et en considérant la somme des $4 \beta_{M}$ d'autre part (courbe 2). On y constate un comportement notablement différent du déplacement radiatif, qui constitue l'amélioration essentielle apportée par la considération de la dégénérescence en $M$. Sur la même figure, nous avons porté le déplacement radiatif obtenu expérimentalement (courbe 3). Nous pouvons y constater que l'accord 


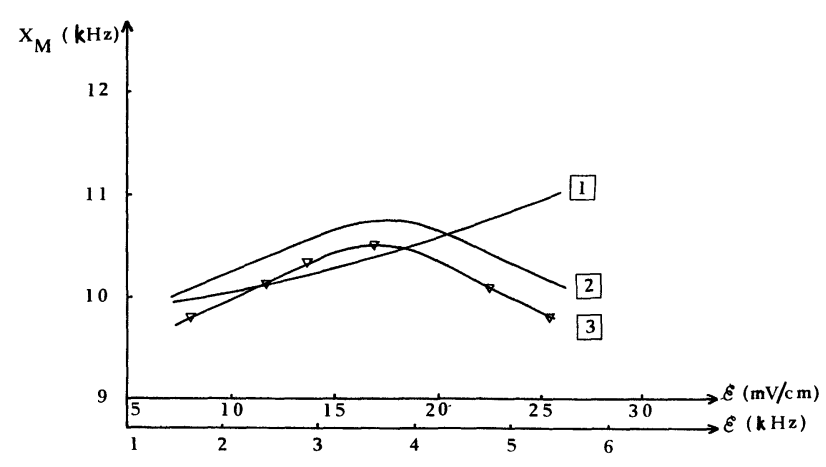

Fig. 4. - Déplacements radiatifs. Courbe $1: \beta$ unique. Courbe 2 : dégénérescence en $M$. Courbe 3 : résultat expérimental.

[Radiative shifts. Curve 1 : single $\beta$. Curve $2: M$ degeneracy. Curve 3 : Experimental result.]

se situe manifestement entre les courbes (2) et (3), le déplacement radiatif passant par un maximum caractéristique.

Une expérience faite avec un grand nombre d'éléments $(n=26)$ ne permet pas d'observer de partie ascendante dans la courbe du déplacement radiatif (Fig. 5), comportement déjà observé par Annabi [9].

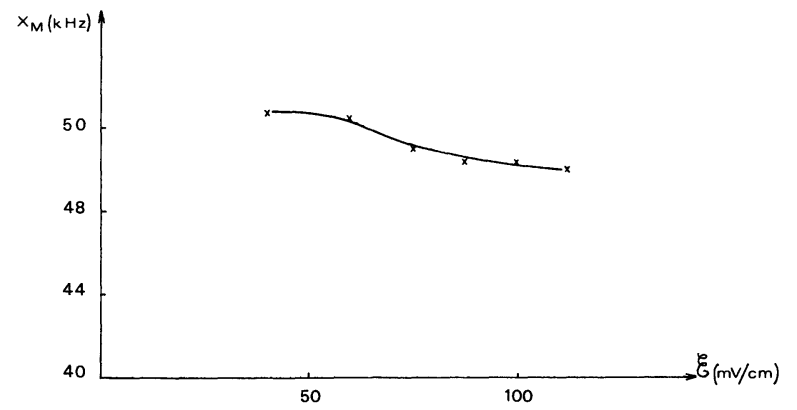

Fig. 5. - Déplacement radiatif expérimental pour $n=26$. [Experimental radiative shift for $n=26$.]
4. Conclusion. - La nouvelle théorie exposée ici constitue, au même titre que celle faite en champ sinusoïdal dans l'article précédent, une approximation du système expérimental. Dans cette mesure, elle n'explique pas de façon parfaite le comportement de la probabilité de transition puisqu'elle prévoit des composantes extérieures que nous n'avons pas observées expérimentalement. Ces composantes prévues par la théorie dérivée de Ramsey sont dues aux harmoniques de modulation en créneau et si elles n'apparaissent pas expérimentalement, c'est précisément parce que le système réel ne reproduit pas de façon exacte cette modulation en créneau. Il n'en reste pas moins que la théorie faite ici rend compte d'un déplacement radiatif de caractère particulier dont le comportement pouvait a priori paraître surprenant puisque lorsque le champ d'irradiation croît, on observe d'abord un déplacement positif, puis un déplacement négatif. Le déplacement positif ne se manifeste que si $n$ est petit, et disparaît lorsque $n$ est grand.

Signalons enfin un aspect particulier des expériences décrites ici et dans les références [1] et [2] : Si, dans l'équation

$$
\frac{\mathrm{d} b_{2}}{\mathrm{~d} t}=\frac{\beta}{i}\left[\mathrm{e}^{i(\Delta+\Omega) t}-\mathrm{e}^{i(\Delta-\Omega) t}\right]
$$

(réf. [ij p. 458) on fait $\omega_{12}=0$, on obtient l'expression décrivant l'évolution d'un système subissant une transition sous l'effet des composantes résonnante et non résonnante du champ d'irradiation. Les deux résonances à $\pm \Omega$ correspondent par exemple à celles observées en résonance magnétique pour 2 directions opposées du champ directeur. Par analogie les oscillations de la probabilité de transition pourraient également être observées sur une transition de fréquence basse, en absence de relaxation, lorsque le temps d'irradiation conduit à une largeur de raie du même ordre de grandeur que la fréquence de transition.

\section{Bibliographie}

[1] Genty, C., Miller, J. J., Theobald, J. G., Revue Phys. Appl. 13 (1978) 457.

[2] Annabi, M., Gillet, D., Revue Phys. Appl. 8 (1973) 1.

[3] Hadeishi, I., Bichel, W. S., Garcia, J. D. and Berry, M. G., Phys. Rev. Lett. 23 (1969) 65.

[4] Pommier, J. et Shermann, J. P., C.R. Hebd. Séan. Acad. Sci. 263 (1966) 830.
[5] Genty, C. et Theobald, J. G., Revue Phys. Appl. 8 (1973) 361.

[6] Chardon, J. C. et al., Revue Phys. Appl. 9 (1974) 961.

[7] Ramsey, N. F., Molecular Beams (Clarendon Press. Oxford) 1956.

[8] Daury, G., Ann. Phys. 4 (1969) 327.

[9] ANnABI, M., Thèse Besançon (1973) A07237. 